\title{
Hybrid Pixels for the Micro-Vertex Detector of the PANDA Experiment
}

\section{Calvo ${ }^{b}$, P. De Remigis ${ }^{b}$, S. Marcello ${ }^{a}$, G. Mazza ${ }^{b}$, M. Mignone ${ }^{b}$, A. Rivetti ${ }^{b}$, R.} Wheadon $^{b}$, L. Zotti ${ }^{* a}$

${ }^{a}$ Università degli Studi di Torino \& INFN Torino

Via Pietro Giuria 1, Torino

Italy

${ }^{b}$ INFN Torino

Via Pietro Giuria 1, Torino

Italy

\begin{abstract}
PANDA is a fixed target experiment with antiproton beam up to $15 \mathrm{GeV} / \mathrm{c}$, that will take place at the future FAIR facility. The PANDA experiment will allow the study of different physics topics, from hadron structure and quark-gluon dynamics to nuclear physics. The Micro-Vertex Detector (MVD) is the detector closest to the interaction point, and it is equipped with pixel and strip detectors. The MVD is designed to reconstruct the secondary vertices of the involved charmed mesons, featuring good spatial resolution, limited material budget, radiation hardness, and PID capability. The peculiar requirement to work in a triggerless environment asks for the capability to handle very high data rates.

The hybrid pixel R\&D program is in its final stage. Its design is based on thin epitaxial sensors and readout chip developed in 130nm CMOS technology. Here the latest pixel detector R\&D achievements are presented.
\end{abstract}

50th International Winter Meeting on Nuclear Physics

23-27 January 2012

Bormio, Italy

\footnotetext{
* Speaker.
} 


\section{Introduction}

The $\bar{P}$ ANDA (AntiProton ANnihilation at DArmstadt) experiment [1] is one of the key projects at the future Facility for Antiproton and Ion Research (FAIR) [2], which is currently under construction at GSI, Darmstadt. The FAIR accelerators will deliver primary proton and ion beams as well as secondary beams of antiprotons or radioactive ions, all with high energy, high intensity and high quality. The new facility FAIR together with PANDA will provide an excellent tool to address fundamental questions on hadronic physics. The antiproton beam of unprecedented intensity and quality in the momentum range from $1.5 \mathrm{GeV} / \mathrm{c}$ up to $15 \mathrm{GeV} / \mathrm{c}$ will be stored in the High Energy Storage Ring (HESR) and will provide access to strange and charm quarks and to a copious production of gluons. The physics program offers a broad range of studies that extends from the investigation of QCD to the test of fundamental symmetries, providing a link between hadronic and nuclear physics [3]. The general PANDA detection concept requires a coverage over nearly the full solid angle, and it is based on two magnetic spectrometers:

- a target spectrometer placed around the interaction region, equipped with a superconducting solenoid for momentum measurement;

- a forward spectrometer, with a large gap dipole magnet, will be used to reconstruct and to identify the high momentum particles emitted at angles of $\pm 5^{\circ}$ and $\pm 10^{\circ}$, in the vertical and horizontal direction respectively.

The combination of the two spectrometers allows the tracking of almost all particles, either those with small momenta going at large angles, or those with high momenta emitted in the forward region due to the Lorentz boost [3].

\section{The Micro Vertex Detector}

The MVD is the innermost part of the central tracking system, and it has to cover polar angles from $3^{\circ}$ up to $150^{\circ}$. The main task of the MVD is a precise measurement of both primary and secondary vertices. The most important requirements of the MVD are:

- Spatial resolution In order to resolve the displaced decay vertices of open-charm states (such as $D^{0}$ and $D^{ \pm}$), the vertex resolution should be better than $100 \mu \mathrm{m}$. At energies close to the threshold the charmed mesons will be strongly forward focused, in this case the best resolution is required along the beam direction (z-direction). Along the $\mathrm{x}$-y direction the resolution should be of a few tens of $\mu \mathrm{m}$.

- Low Material Budget The MVD material choice is dictated by the compromise between the system feasibility and the material budget, in order to avoid the deterioration of the tracking performances and to allow an efficient operation of the electromagnetic calorimeter. The total material budget stays below $10 \%$ in unit of radiation length and it is rather isotropic.

- Time resolution The $\bar{P} A N D A$ choice of continuous data collection without external trigger is a stringent requirement for the readout concept. The critical aspect of the MVD data extraction is related to the high interaction rates, which will exceed $10^{7} \bar{p}-p$ annihilations 
per second. For this reason the time resolution should be better than $10 \mathrm{~ns}$ in order to perform particle tracking in a triggerless environment.

- Radiation tolerance In the MVD the expected fluence is strongly peaked in the forward direction and it has been estimated to be of the order of $10^{14} \mathrm{n}_{1 \mathrm{MeVeq}} \mathrm{cm}^{-2}$ per 10 years of data taking and a 50\% duty cycle ( $\overline{\mathrm{p}}-\mathrm{p}$ annihilations). The expected total ionizing dose is below $100 \mathrm{kGy}$.

The momentum resolution of the global tracking can be improved by roughly a factor two thanks to the MVD that defines important constraints to the tracking algorithms, facilitating the particle momentum determination. The momentum resolution achievable by the overall tracking will be better than $2 \%$ in the angular polar range between $20^{\circ}$ and $140^{\circ}$, and better than $3 \%$ between $12^{\circ}$ and $20^{\circ}$.

In addition the MVD can contribute to the overall particle identification, thanks to the detector capability to perform energy loss measurements. As shown in Fig.11, the MVD can contribute to the global PID decision up to a momentum of $500 \mathrm{MeV} / \mathrm{c}$ for kaons and up to $1 \mathrm{GeV} / \mathrm{c}$ for protons, while no additional information can be given for pions, muons and electrons.

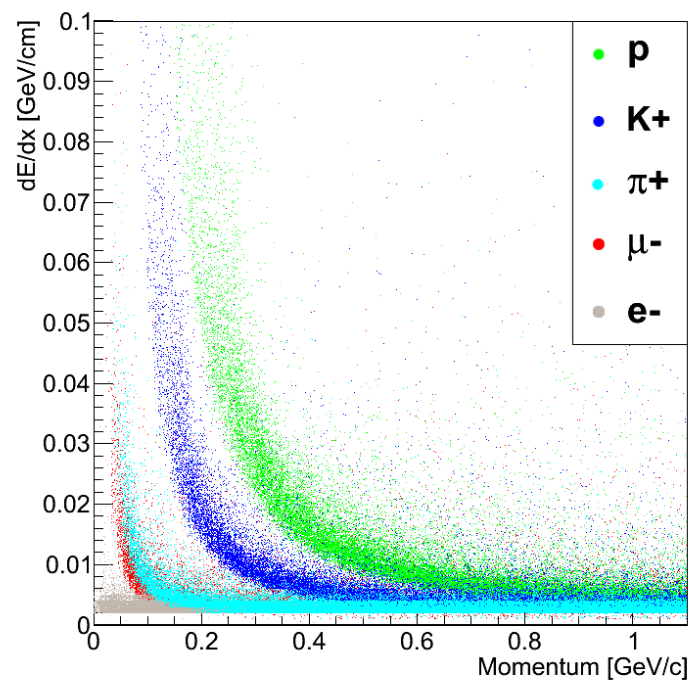

Figure 1: Specific energy loss distribution in the MVD as a function of the momentum.

The MVD is divided into a barrel and a forward system, as shown in Fig. 2. The first layer is located $2.5 \mathrm{~cm}$ far from the interaction point, while the central tracker limits the outer maximum diameter of the MVD to $30 \mathrm{~cm}$. Along the beam direction the MVD extends roughly $\pm 23 \mathrm{~cm}$ with respect to the nominal interaction point. The barrel is composed of 4 coaxial cylindrical layers; the forward part is formed by 6 layers of circular shape. The two innermost barrel layers and the forward disks will be equipped by silicon hybrid pixels, because of their precise space point measurements, and also to cope with the high occupancy close to the interaction region. Double-side silicon micro-strips will be used in the outer barrel layers and as radial components on the two last 


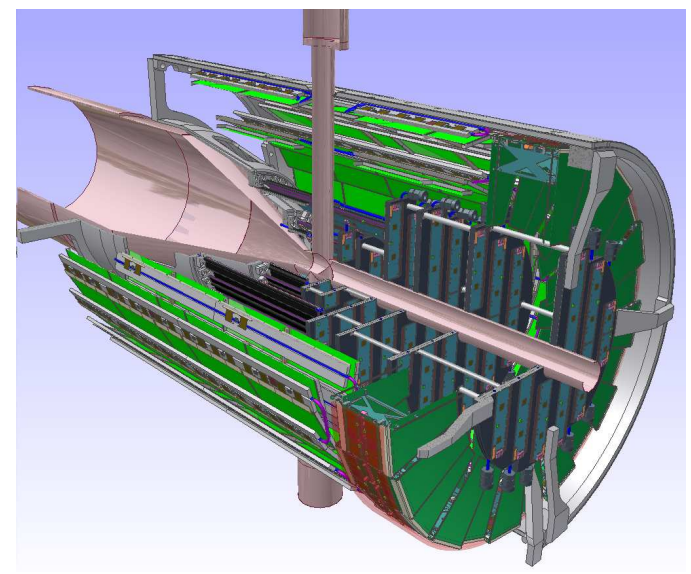

Figure 2: The MVD layout.

disks. The total amount of readout channels will be $\sim 10^{7}$ for the hybrid pixels and $\sim 2 \cdot 10^{5}$ double side micro-strips.

\section{Hybrid Pixel Detector}

A scheme of the PANDA hybrid pixel is shown in Fig. 3 . The hybrid pixel detector is composed of reverse-biased epitaxial pixel sensor connected by solder-bump bonding to the frontal readout cell of the ASIC developed in 130nm CMOS technology. This assembly will be glued on a carbon foam layer, in order to improve the heat dissipation towards the cooling system.

The high granularity request and the simulated benchmark physics channels (as explained in de-

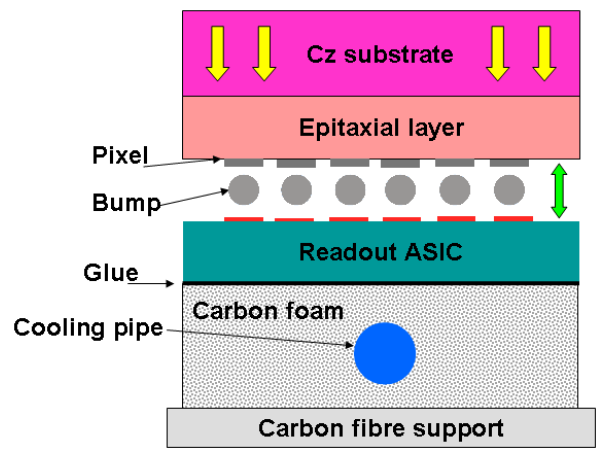

Figure 3: Schematic view of the hybrid pixel for $\bar{P} A N D A$.

tails in [3]) suggest a pixel size of $100 \mu \mathrm{m} \times 100 \mu \mathrm{m}$ [П]. The high radiation hardness of the epitaxial silicon sensor, the possibility to obtain thinned pixel sensor (removing the Czochralski substrate on which the epitaxial silicon layer grows), and the effort to reduce the material budget make the choice of a $100 \mu \mathrm{m}$ thick sensors the best compromise together with the possibilities to perform energy loss measurements and to have a good signal to noise ratio. 


\subsection{Sensor Technology and R\&D}

The $\bar{P} A N D A$ sensor will be made of epitaxial silicon layer with high resistivity (a few $\mathrm{k} \Omega \cdot \mathrm{cm}$ ), grown on a Cz substrate with a lower resistivity $(10-20 \mathrm{~m} \Omega \cdot \mathrm{cm})$. Most of this substrate is removed starting from the side opposite to the epitaxial layer.

Displacement damage tests of several epitaxial diodes have been performed with neutrons, from the nuclear reactor TRIGA MARK II of Pavia (LENA laboratory), in order to study the behavior of the depletion voltage and of the leakage current as a function of the annealing time.

The samples feature different values of the resistivity and of the epitaxial layer thickness, see Tab.1. Before and after irradiation $\mathrm{C}-\mathrm{V}$ and $\mathrm{I}-\mathrm{V}$ measurements were performed. The reached equivalent fluence was $1.5 \cdot 10^{14} \mathrm{n}_{1 \mathrm{MeVeq}} \mathrm{cm}^{-2}$. After the irradiation an annealing phase at $60^{\circ}$ started; during this period additional measurements of the $\mathrm{C}-\mathrm{V}$ and $\mathrm{I}-\mathrm{V}$ were performed.

The full depletion voltages $\left(V_{\text {dep }}\right)$ of the studied samples before the irradiation are reported in Tab.1. As expected the $V_{\text {dep }}$ decreases for the high resistivity diodes, since the depletion voltage is inversely proportional to the resistivity, while the $V_{\text {dep }}$ increases with the thickness.

In Fig. $\theta$ the trend of the $V_{\text {dep }}$ during the annealing phase is shown. The values reported at time zero represent the $V_{\text {dep }}$ immediately after irradiation and before the annealing phase. The higher values of the $V_{\text {dep }}$ in the middle and high resistivity samples are explained by a reverse annealing effect occurred before the $60^{\circ}$ temperature phase. In the low resistivity specimen the type inversion occurred only after few days of $60^{\circ}$ temperature phase.

\begin{tabular}{|l|c|c|}
\hline Diodes & Resistivity $[\Omega \cdot \mathrm{cm}]$ & Full Dep. Voltage [V] \\
\hline Epi-50 & $4060(\mathrm{HR})$ & $4.35 \pm 0.06$ \\
\hline Epi-75 & $4570(\mathrm{HR})$ & $5.6 \pm 0.1$ \\
\hline Epi-100 & $4900(\mathrm{HR})$ & $5.9 \pm 0.2$ \\
\hline Epi-50 & $3114(\mathrm{MR})$ & $4.91 \pm 0.07$ \\
\hline Epi-75 & $3224(\mathrm{MR})$ & $8.2 \pm 0.3$ \\
\hline Epi-100 & $3609(\mathrm{MR})$ & $10.4 \pm 0.6$ \\
\hline Epi-75 & $461(\mathrm{LR})$ & $42.6 \pm 1.3$ \\
\hline
\end{tabular}

Table 1: Pre-irradiation full depletion voltage values. HR= High resistivity, MR=Middle Resistivity and LR=Low Resistivity.

The damage parameter $\alpha=\Delta J / \phi$ (where $\Delta J$ is the diode volume current difference before and after irradiation, and $\phi$ is the equivalent fluence), ranges between 5 and $8 \cdot 10^{-17} \mathrm{~A} / \mathrm{cm}$. A leakage current of $13 \mathrm{nA} /$ pixel has been extrapolated from the diode volume current taking into account the foreseen pixel volume of $100 \times 100 \times 100 \mu \mathrm{m}^{3}$. Since the frontend electronics is able to compensate the leakage current up to $60 \mathrm{nA} /$ pixel this value is acceptable.

In Fig. [ the first full size sensor prototype, that is foreseen to be p-in-n, is shown. On the left side the wafer, with the pixel sensors and the diagnostic structures at the edge, is reported. On the right side a partial view of the $100 \mu \mathrm{m} \times 100 \mu \mathrm{m}$ pixel matrix is visible, the white circles are the pads for the bumps. Along the columns the pitch is $100 \mu \mathrm{m}$, meanwhile along the rows it is alternatively $50 \mu \mathrm{m}$ and $150 \mu \mathrm{m}$. 


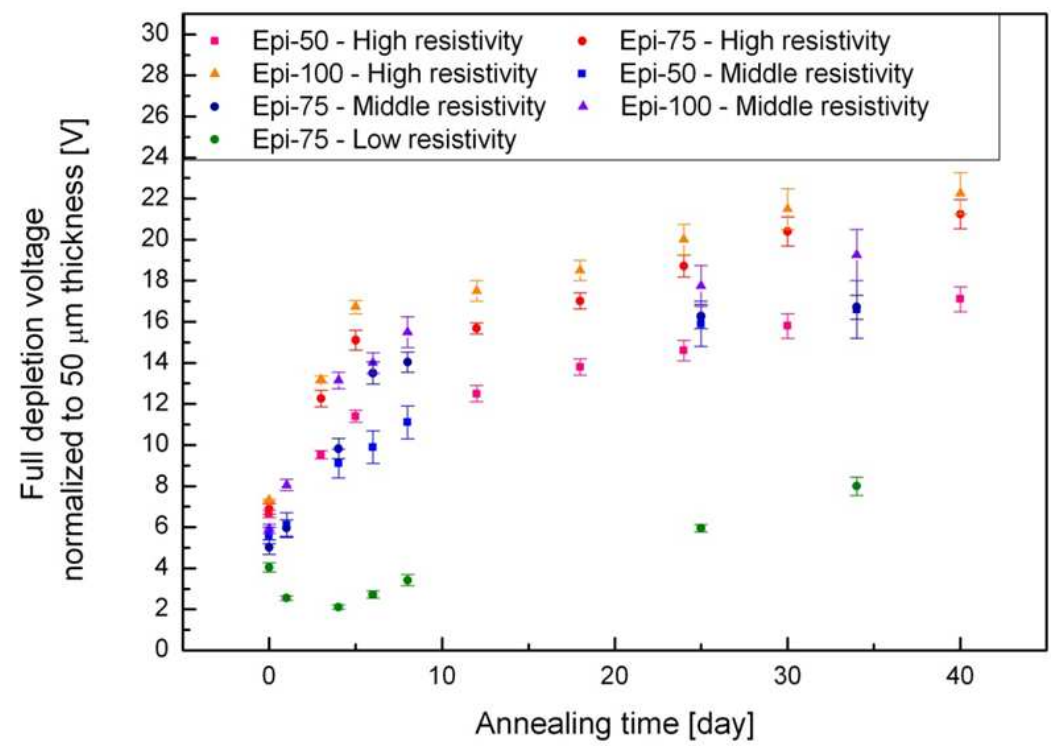

Figure 4: Behavior of the $V_{\text {dep }}$ as a function of the annealing time [5].

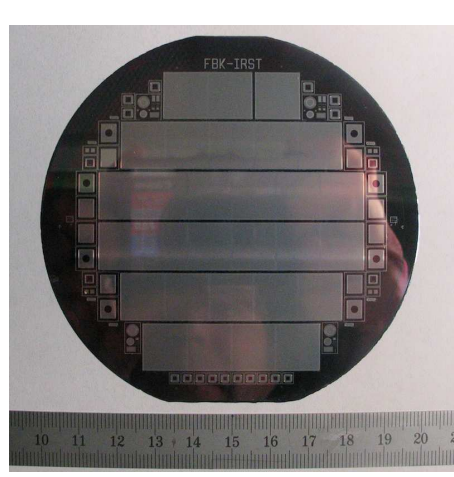

(a) Picture of a $\bar{P} A N D A$ wafer.

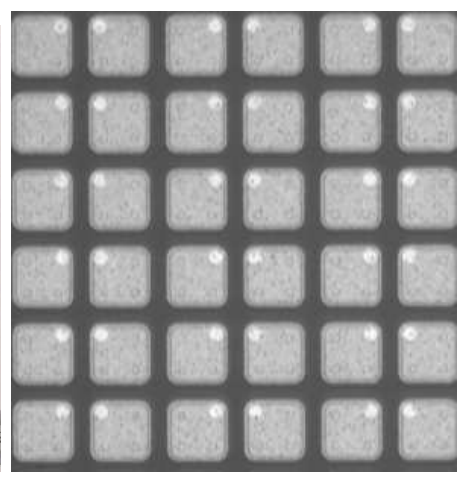

(b) Part of a pixel matrix. The white circles at one corner are the pads for the bumps.

Figure 5: Pictures of the first designed full size sensor prototype.

\subsection{Readout ASIC in CMOS 130 nm: ToPix}

The MVD requirements dictate the specifications for the readout electronics. For each hit a simultaneous position, time and charge digital information have to be provided by the ASIC. The electronics has been designed with selectable input polarity to be able to use both p-in-n and n-in-n sensors. The $\bar{P} A N D A$ triggerless operation requires a higher system clock of $155.52 \mathrm{MHz}$ (time resolution of $\sim 6 \mathrm{~ns}$ and $1.85 \mathrm{~ns} \mathrm{rms}$ ). The electronics will be able to handle an expected maximum event rate of about $6 \cdot 10^{6}$ hits $\mathrm{s}^{-1} \mathrm{~cm}^{-2}$. The main ASIC specifications are:

- readout cell size of $100 \mu \mathrm{m} \times 100 \mu \mathrm{m}$, due to the geometry of the pixel sensors to whom the readout cells must be individually connected through bump bonding. The chip size is $11.6 \times 14.8 \mathrm{~mm}^{2}$; 
- input range up to $50 \mathrm{fC}$;

- noise level $<0.032 \mathrm{fC}\left(200 \mathrm{e}^{-}\right)$;

- measurement of $\mathrm{dE} / \mathrm{dx}$ with a resolution of 12-bit with the Time over Threshold (ToT) technique;

- power dissipation $<800 \mathrm{~mW} / \mathrm{cm}^{2}$;

- radiation tolerance up to a $10 \mathrm{Mrad}$ in 10 years (50\% duty cycle);

- tolerance up to a fluence of $10^{14} \mathrm{n}_{1 \mathrm{MeVeq}} \mathrm{cm}^{-2}$ in 10 years (50\% duty cycle);

Fig.6 shows the analog readout part of a pixel cell. The detector is modeled as a variable current source representing the actual signal, a constant leakage current source and a detector capacitance. The architecture is based on the Time over Threshold (ToT) technique, which allows a good linearity over a wide charge range even with a saturated amplifier. The current from the detector is integrated in a Charge Sensitive Amplifier (CSA), charging the feedback capacitor $C_{f}$; the ToT technique consists in reconstructing the injected charge by measuring the time which is necessary to discharge the capacitor through a constant current feedback. Since an AC coupling with the sensor is not possible due to strict space constraints, a second feedback network is used to compensate the leakage current. It consists in a low pass differential amplifier, which senses the difference between the CSA output and the reference baseline voltage; the differential amplifier drives a current source which injects the compensating current in the input node of the CSA. The CSA output is then compared with a user defined threshold value, which is fixed externally but it can be finetuned on a pixel by pixel basis with a 5-bit DAC. The comparator generates a pulse whose width, the Time over Threshold, is proportional to the amplitude of the signal [6].

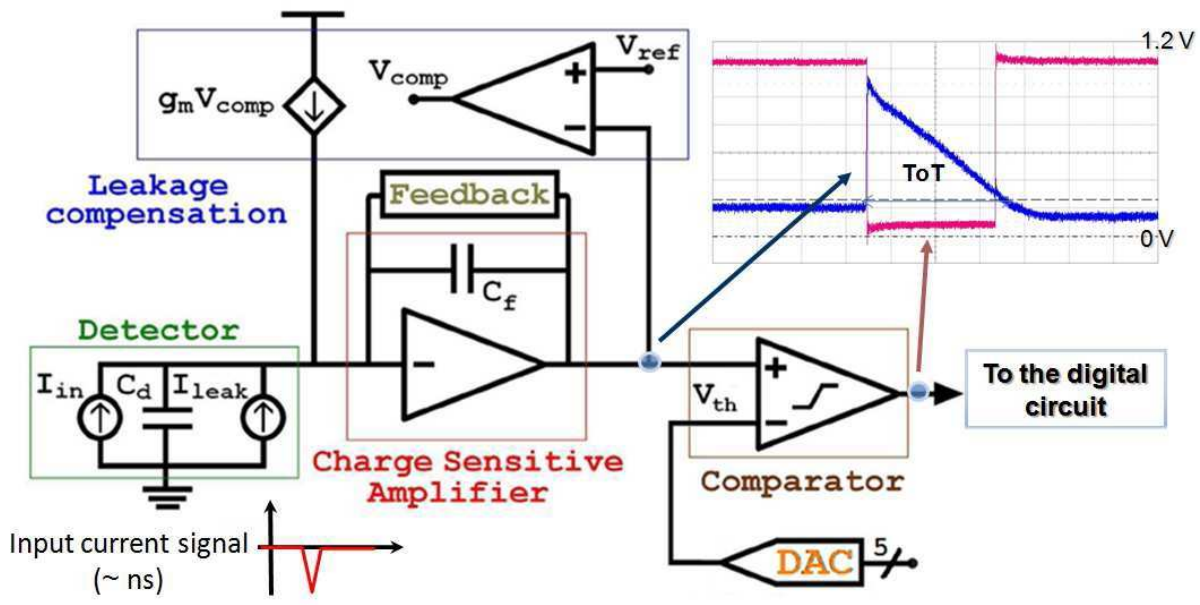

Figure 6: Analogue readout part of a pixel cell.

\subsubsection{The third prototype: ToPix 3}

The third prototype of the ToPix chip has been designed and tested [0]. It covers a die area of $4.5 \times 4 \mathrm{~mm}^{2}$ and contains 640 pixel readout cells, arranged in $2 \times 32$ and $2 \times 128$ double columns. 
The 128 cells columns have been folded in four 32-cells sub-columns in order to simplify the die handling. Bump bonding pads have been used in the pixel cells for direct connections to the detector. All the flip-flops in the configuration registers are fully static, and are SEU protected using the triple-redundancy. The end of the column control logic has been implemented. The analogue and digital part occupy the same area of $50 \times 100 \mu \mathrm{m}^{2}$.

The ToPix 3 prototype has been tested on a bench at the INFN-Torino workshop to verify its performances. The experimental setup foresees a testing board housing ToPix 3 and a Xilinx evaluation board equipped with the Virtex 6 FPGA. A LabView software is used as acquisition system. An internal calibration system is implemented on the chip; then it is possible to stimulate each pixel using a voltage input signal through a capacitor featuring a nominal capacitance of $36 \mathrm{pF}$.

Fig.77 shows the reconstructed signal shape as a function of the increasing input charge values. The fast rising edge and the slow discharge at constant current can be observed from the obtained shape. In the left part of Fig.8 the charge to time overall transfer function for the full charge input range is

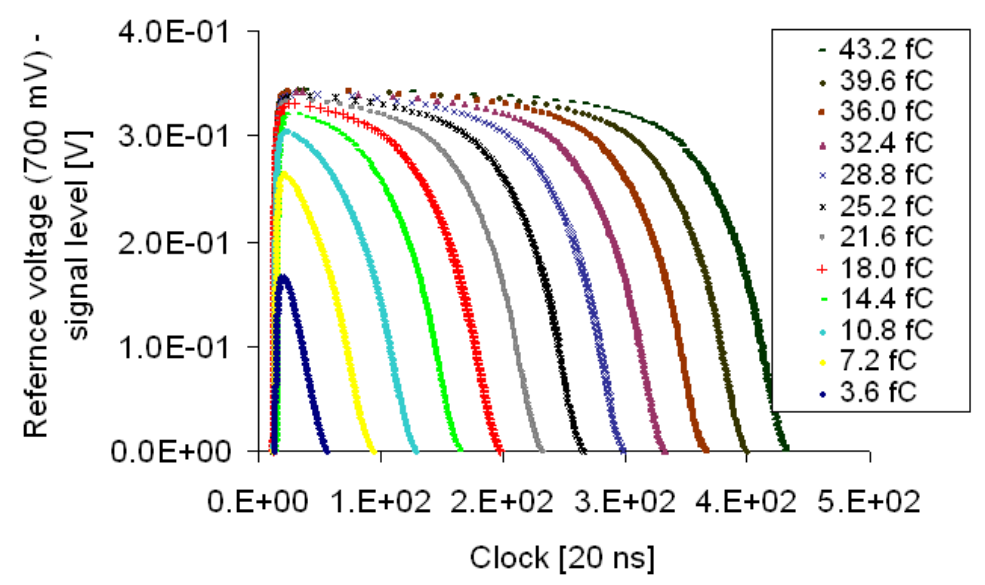

Figure 7: Reconstructed signal shape obtained with a threshold scan and different input charge values.

shown, and a good linearity is obtained even when the signal is saturated, as shown in Fig. 7. On the right part of Fig 8 the trends of the ToT output as a function of the input charge (up to $6 \mathrm{fC}$ ) is plotted, the linearity is good down to almost $1 \mathrm{fC}$.

The pixels baseline distribution is shown in Fig.9, before and after the correction. Before the correction, the baseline dispersion was $5 \mathrm{mV}$ corresponding to $473 \mathrm{e}^{-}$equivalent charge dispersion. Using the on-pixel 5-bit DACs it was possible to correct the baseline values, obtaining a final dispersion of $0.65 \mathrm{mV}$ corresponding to less than $62 \mathrm{e}^{-}$.

Total Ionizing Dose test has been performed on two chips using the X-ray irradiation facility at CERN with a dose rate of $400 \mathrm{rad} / \mathrm{s}$ for a total accumulated dose of $\sim 30 \mathrm{Mrad}$. After irradiation an annealing phase at $80^{\circ}$ has been followed. In Fig 10 the experimental points corresponding to the mean value of the measured baseline distributions are reported. The baseline mean values of the two tested chips both present a similar trend during the irradiation and the annealing phase. The two 32-cell columns at the edge of the readout cell matrix are not included in the evaluated average since their behavior during the irradiation is still under investigation. An average baseline 

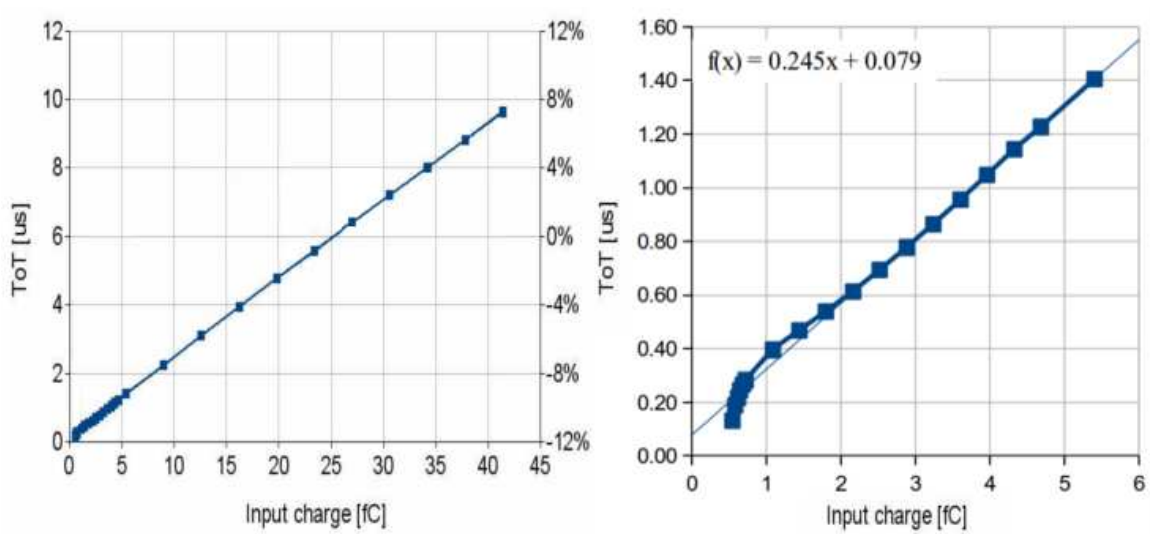

Figure 8: ToT linearity performance. On the left the transfer function in the full range. On the right the transfer function for a nominal feedback current ( $0.6 \mathrm{fC}$ charge).
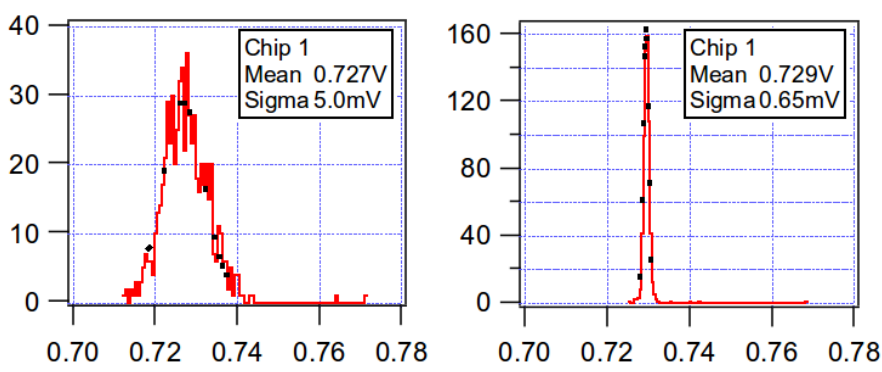

Extrapolated baseline (V) Extrapolated baseline (V)

Figure 9: Baseline distribution before (left) and after correction (right).

dispersion less than $1.4 \%$ has been observed. Besides in both the prototypes the initial average baselines are restored during the annealing phase.

\section{Conclusions}

The $\bar{P} A N D A$ hybrid pixel R\&D phase is in its final stage. The sensor technology choice has been demonstrated to be feasible. The third ToPix prototype characterization is ongoing, the preliminary test results show a satisfactory electrical performance.

\section{Acknowledgments}

The authors would like to thank F. Faccio for the use of the X-Ray source at CERN.

\section{References}

[1] PANDA collaboration, Technical Progress Report FAIR STI/QCD-PAC (2005)

[2] GSI Helmholtzzentrum für Schwerionenforschung, FAIR - An International Accelerator Facility for Beams of Ions and Antiprotons, Baseline technical Report (2006)

[http://www.gsi.de/fair/reports/btr.html] 

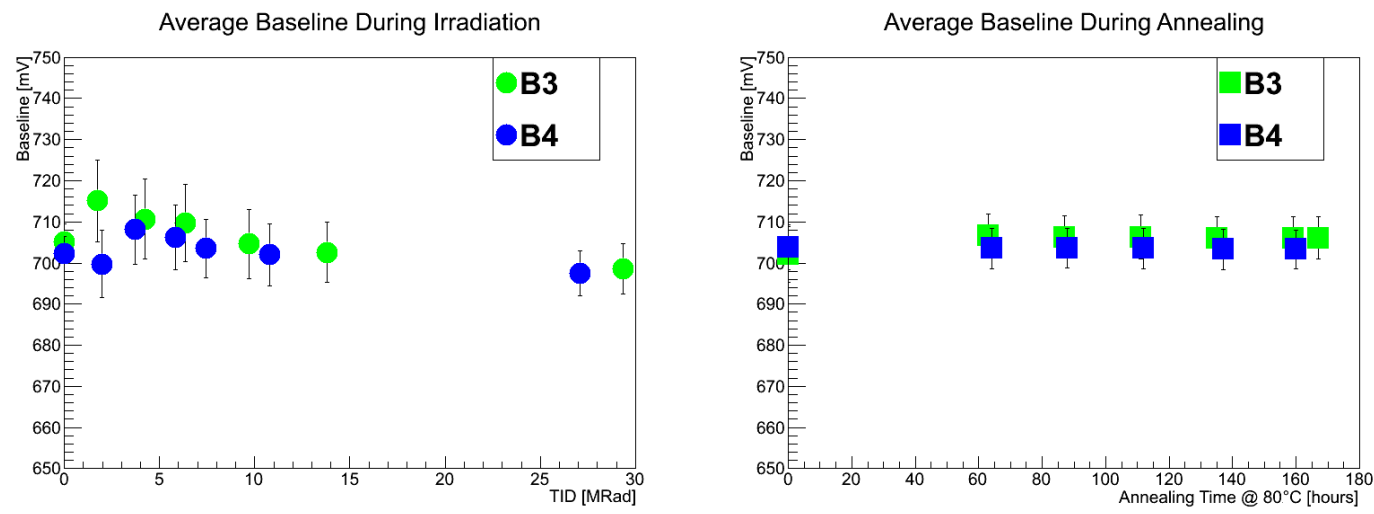

(a) Average baseline trends during the X-ray irradiation. (b) Average baseline trends during the $80^{\circ}$ temperature phase.

Figure 10: Trends of the average baseline of two tested chips.

[3] PANDA collaboration. Physics Performance Report for PANDA - Strong Interaction Studies with Antiprotons., Physics Report (2009) [arXiv: $0903.3905 v 1]$

[4] René Jäkel, Resolution Studies for the Micro Vertex Detector of the PANDA Experiment and the Reconstruction of Charmed Mesons for Specifc Hadronic Channels, PhD thesis, TU Dresden (2009).

[5] Andrea Tengattini, Radiation damage effects on epitaxial silicon devices for the PANDA experiment, Master Thesis, University of Turin (2010).

[6] Thanushan Kugathasan, Low-Power High Dynamic Range Front-End Electronics for the Hybrid Pixel Detectors of the PANDA MVD, PhD Thesis, University of Turin (2010)

[7] G. Mazza et al. A CMOS 0.13 um Silicon Pixel Detector Readout ASIC for the PANDA experiment, Journal of Instrumentation (2012) [doi:10.1088/1748-0221/7/02/C02015] 